\title{
A!
}

This is an electronic reprint of the original article.

This reprint may differ from the original in pagination and typographic detail.

\author{
Bhattacharya, Ankita; Islam, SK Firoz
}

\section{Photoinduced spin-Hall resonance in a k3 -Rashba spin-orbit coupled two-dimensional hole system}

Published in:

Physical Review B - Condensed Matter and Materials Physics

DOI:

10.1103/PhysRevB.104.L081411

Published: 15/08/2021

Document Version

Publisher's PDF, also known as Version of record

Please cite the original version:

Bhattacharya, A., \& Islam, SK. F. (2021). Photoinduced spin-Hall resonance in a k3 -Rashba spin-orbit coupled two-dimensional hole system. Physical Review B - Condensed Matter and Materials Physics, 104(8), 1-6.

[081411]. https://doi.org/10.1103/PhysRevB.104.L081411

This material is protected by copyright and other intellectual property rights, and duplication or sale of all or part of any of the repository collections is not permitted, except that material may be duplicated by you for your research use or educational purposes in electronic or print form. You must obtain permission for any other use. Electronic or print copies may not be offered, whether for sale or otherwise to anyone who is not an authorised user. 


\title{
Photoinduced spin-Hall resonance in a $\boldsymbol{k}^{3}$-Rashba spin-orbit coupled two-dimensional hole system
}

\author{
Ankita Bhattacharya ${ }^{1, *}$ and SK Firoz Islam $\odot^{2, \dagger}$ \\ ${ }^{1}$ Institute of Theoretical Physics, Technische Universität Dresden, 01062 Dresden, Germany \\ ${ }^{2}$ Department of Applied Physics, Aalto University, P.O. Box 15100, FI-00076 Aalto, Finland
}

(Received 12 June 2021; revised 18 August 2021; accepted 18 August 2021; published 30 August 2021)

\begin{abstract}
We study the band structure modulation and spin-Hall effect of an irradiated two-dimensional heavy-hole system with $k^{3}$-Rashba spin-orbit coupling. We find that the band structure becomes anisotropic under the illumination by the light. Most remarkably, in the presence of linearly polarized light a pair of additional spin-degeneracy points, apart from the $\Gamma$ point, emerge in the energy dispersion. The locations of these points are solely determined by the amplitude of the incident light. If this degeneracy occurs around the Fermi level, the spin-Hall conductivity exhibits a resonance. Away from the degeneracy points, the light rotates the average spin polarization. The possible effects of $k^{3}$-Dresselhaus spin-orbit coupling are also discussed.
\end{abstract}

DOI: 10.1103/PhysRevB.104.L081411

Introduction. Recent years have witnessed growing interest in the interaction of light with electronic systems, especially after the discovery of light-induced topological phase transitions [1-6] (Floquet topological insulator), followed by several experiments [7-10]. Such an interaction can also be used to manipulate the spin- and valley-selective transport in Dirac materials [11-14] and transition-metal dichalcogenides $[15,16]$. There have been extensive works on phototunable Weyl nodes [17-21], the $0-\pi$ transition in Josephson junctions [22,23], and Floquet engineering of Majorana modes [24-27]. Interfacial chiral modes have also been predicted in threefold topological semimetals [28], by controlling the phase of the light. More recently, higher-order topological insulators [29-31] have also been predicted in different irradiated materials.

On the other hand, two-dimensional systems with strong spin-orbit interactions have become promising test beds for future spintronics development. The Rashba spin-orbit coupling (RSOC) [32,33] in two-dimensional (2D) fermionic systems arises due to the lack of structural inversion symmetry in the quantum well of semiconductor heterojunctions, which removes spin degeneracy in the absence of a magnetic field. Several experiments have confirmed that the strength of RSOC can be significantly enhanced by applying a gate voltage across the quantum well [34-36]. There are two types of RSOC, namely linear and cubic in momentum $(k)$. Typical materials which exhibit $k$-linear RSOC are indiumbased compounds such as InAs, GaInAs/GaAlAs structures, and II-VI semiconductor compounds. On the other hand, $k^{3}$ RSOC arises due to the structural bulk inversion asymmetry for heavy holes in the quantum well of III-V semiconductor heterojunctions. The spin-Hall effect (SHE) [37-40] is one of the exciting phenomena in a 2D electron/hole gas with RSOC, in which electrons or holes with opposite spin are accumulated at opposite transverse edges normal to the ap-

\footnotetext{
*ankita.bhattacharya@tu-dresden.de

†firoz.seikh@aalto.fi
}

plied in-plane electric field. The spin-Hall conductivity (SHC) was predicted to exhibit a universal constant value in $2 \mathrm{D}$ electron gas (2DEG) with $k$-RSOC [38]. However, further study revealed that the disorder-induced vortex correction strongly suppresses the SHC in such systems [41,42]. The SHC in a $k^{3}$-RSOC 2D heavy-hole gas (2DHG) was also investigated and found to be robust to the vortex correction [43], for which this system has an edge over 2DEG with RSOC. Moreover, the strong spin-orbit coupling and weak hyperfine interaction in a heavy-hole gas allows low-power electrical manipulation of spin [44-46]. Another study on the SHE in a 2D hole system has revealed that the SHC strongly depends on the strength of RSOC [47], which is in contrast to the corresponding electron system. The magnetic-field-dependent transport signatures (Shubnikov-de Hass oscillation and quantum-Hall conductivity) [48-53] have been considered in both systems, revealing the roles of RSOC. Recently, a series of theoretical works on spin-related phenomena in $2 \mathrm{DHG}$ in the presence of a weak magnetic field have been studied by the Culcer group [54-57]. Moreover, the search for materials or mechanisms to achieve giant or large SHC [58-60] continues to be an active topic in the field of spintronics. It is noteworthy that the SHE is one of the key signatures of spin-Hall edge modes in the quantum-spin-Hall liquid discovered in 2D Dirac materials with spin-orbit coupling $[61,62]$. However, due to the restricted number of spin-Hall edge modes and unavoidable bulk effects, the enhancement of SHC is challenging there. Moreover, from an experiment perspective, semiconductor compounds are still a much more reliable platform than $2 \mathrm{D}$ Dirac materials.

The study of the interplay of the light with $k$-RSOC in 2DEG has been initiated by Ojanen et al. [63], predicting chiral edge states and out-of-plane magnetization. Very recently, the combined effects of light and a real magnetic field have also been studied in this system [64]. However, to the best of our knowledge, the effects of light on $2 \mathrm{DHG}$ in the presence of $k^{3}$-RSOC have not been addressed yet.

In this Letter, we consider the interaction of linearly polarized light with $2 \mathrm{DHG}$ in the presence of $k^{3}$-RSOC. The 


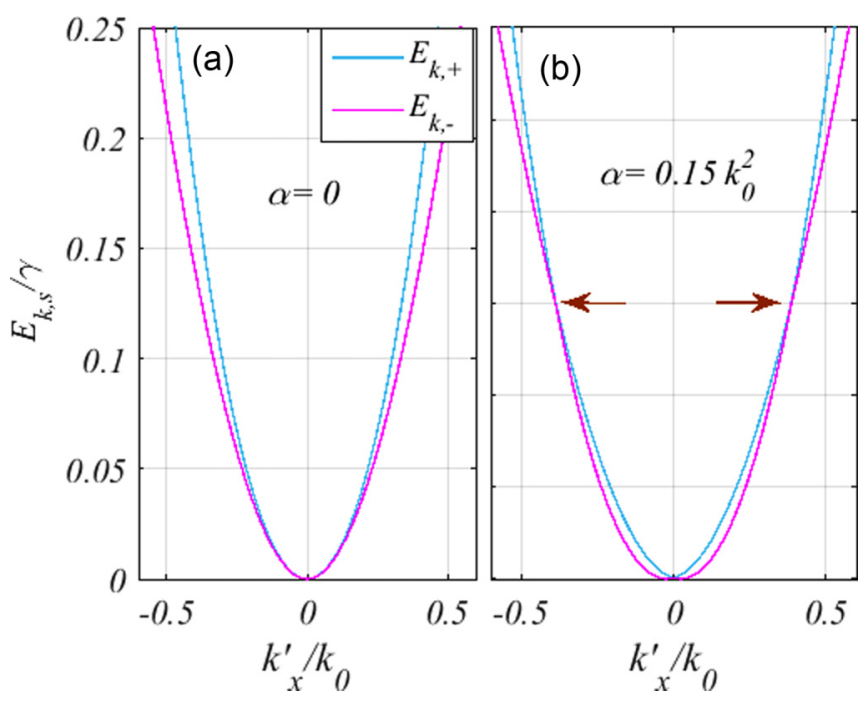

FIG. 1. Energy dispersions are shown for (a) $\alpha=0$ and (b) $\alpha=$ $0.15 k_{0}^{2}$. Here, the momentum axis is rotated by $\pi / 4\left(k_{x}^{\prime}=k_{x} / \sqrt{2}\right)$ to capture the degeneracy points which are shown by two arrows. The energy axis is normalized by $\gamma=\hbar^{2} k_{0}^{2} / 2 m^{*}$, where $k_{0}$ is a typical wave vector, corresponding to standard hole density. The strength of $\mathrm{RSOC}$ is taken to be $\beta k_{0}^{3}=0.4$.

irradiated band structure is found to be anisotropic. Instead of out-of-plane magnetization, linearly polarized light rotates the average spin polarization in the plane of 2DEG. Most remarkably, we reveal that there exist two spin-degeneracy points in the band dispersion, which give rise to a resonance in the SHC. We also discuss the possible effects of the $k^{3}$ Dresselhaus spin-orbit coupling (DSOC) on SHC.

Model Hamiltonian and energy spectrum. We start with a 2DHG in the presence of $k^{3}$-RSOC. The hole dynamics at the top of the valence band in III-V semiconductor quantum wells is generally described by the $4 \times 4$ Luttinger Hamiltonian [65]. Due to the large splitting between the heavy-hole (HH) states $(|3 / 2, \pm 3 / 2\rangle)$ and the light-hole (LH) states $(|3 / 2, \pm 1 / 2\rangle)$ and to the higher density of states of $\mathrm{HH}$ states, the contribution in the transport properties comes predominantly from the $\mathrm{HH}$ states close to the Fermi energy. Thus the model system can be described by a $2 \times 2$ Hamiltonian by projecting onto the $\mathrm{HH}$ states. The single-particle Hamiltonian of this system is given by $[47,66] H=\hbar^{2} k^{2} / 2 m^{*}+H_{R}$, where the RSOC is given by $H_{R}=(\beta / 2 i)\left(k_{-}^{3} \sigma_{+}-k_{+}^{3} \sigma_{-}\right)$with $k_{ \pm}=$ $k_{x} \pm i k_{y}$ and $\sigma_{ \pm}=\sigma_{x} \pm i \sigma_{y}$. Here, $\mathbf{k}=\left(k_{x}, k_{y}\right)$ are the 2D momentum operators whereas $\sigma=\left(\sigma_{x}, \sigma_{y}\right)$ are the two of the three Pauli spin matrices which act on the total angular momentum states with spin projection $\pm 3 / 2$, respectively. The strength of the RSOC and the effective mass of the hole are denoted by $\beta$ and $m^{*}$, respectively. The spin-dependent energy spectrum can be written as $E_{k, s}=\hbar^{2} k^{2} / 2 m^{*}+s \beta|k|^{3}$, where $s= \pm$ stands for two different spin branches. The dispersion is shown in Fig. 1(a). It is to be noted that this model Hamiltonian is valid when the wave number $k \leqslant \hbar^{2} / 2 m^{*} \beta$.

Effects of irradiation. Let us now consider that the system is subjected to an external time-dependent periodic perturbation in the form of irradiation (light), propagating along the $z$ direction. The light field is described by a vector poten- tial $\mathbf{A}(t)=A_{0}\left[\sin (\Omega t), \sin \left(\Omega t+\phi_{0}\right)\right]$, where $A_{0}$ is the field amplitude, $\Omega$ is the frequency of the irradiation, and $\phi_{0}$ is the phase. Here, we consider that the light wavelength is large compare to the lattice constant, for which we can safely ignore the spatial dependence of the light field. The lightinduced vector field can be included into the Hamiltonian as $\mathbf{k} \rightarrow \mathbf{k}+e \mathbf{A}(t) / \hbar$, where $e<0$ is the electron charge. To solve the periodically perturbed Hamiltonian, we can use the Floquet theory [67], which states that such a perturbed Hamiltonian exhibits a complete set of orthonormal solutions of the form $\psi(t)=\phi(t) e^{-i E t / \hbar}$ with $\phi(t)=\phi(t+\mathcal{T})$ being the corresponding Floquet states and $\mathcal{T}$ is the period of the field. Here, $E$ denotes the Floquet quasienergy. The timedependent Schrödinger equation corresponding to the Floquet states yields the Floquet eigenvalue equation as $H_{F} \phi(t)=$ $E \phi(t)$ with $H_{F}=H(t)-i \hbar \partial_{t}$. The Floquet states can be further expressed as $\phi(t)=\sum_{n} \phi_{n}(t) e^{i n \Omega t}$, where $n$ is the Fourier component or Floquet sideband index. By diagonalizing the Floquet Hamiltonian in the basis of Floquet sidebands $n$, one can obtain the Floquet quasienergy spectrum. On the other hand, an effective Hamiltonian can be obtained in the high-frequency limit following the Floquet-Magnus expansion in a power of $1 /(\hbar \Omega)$ as $H_{F} \simeq H+H_{F}^{(0)}+H_{F}^{(1)}+\cdots$, where $H_{F}^{(0)}=-\alpha \beta \cos \phi_{0}\left(\sigma_{x} k_{x}+\sigma_{y} k_{y}\right)$ with $\alpha=3\left(e A_{0} / \hbar\right)^{2}$ and $H_{F}^{(1)}=\left[H_{-}, H_{+}\right] / \hbar \Omega$. Here,

$$
H_{m}=\frac{1}{\mathcal{T}} \int_{0}^{\mathcal{T}} V(t) e^{-i m \Omega t} d t
$$

with $m= \pm$. Here, $V(t)=\mathbf{h}(\mathbf{t}) \cdot \sigma$, which for linearly polarized light $\left(\phi_{0}=0\right)$ simplifies to

$$
\begin{aligned}
h_{x}(t)= & \beta\left(e A_{0} / \hbar\right)\left[3\left(k_{y}^{2}-k_{x}^{2}-2 k_{x} k_{y}\right) \sin (\Omega t)\right. \\
& \left.-2\left(e A_{0} / \hbar\right)^{2} \sin ^{3}(\Omega t)+3\left(e A_{0} / \hbar\right) k_{x} \cos (2 \Omega t)\right]
\end{aligned}
$$

and

$$
\begin{aligned}
h_{y}(t)= & \beta\left(e A_{0} / \hbar\right)\left[3\left(k_{x}^{2}-k_{y}^{2}-2 k_{x} k_{y}\right) \sin (\Omega t)\right. \\
& \left.-2\left(e A_{0} / \hbar\right)^{2} \sin ^{3}(\Omega t)+3\left(e A_{0} / \hbar\right) k_{y} \cos (2 \Omega t)\right] .
\end{aligned}
$$

The high-frequency limit in our case is described as $\left(\gamma, \beta k_{0}^{3}\right.$, $\left.e A_{0} \hbar k_{0} / m^{*}, 3 e A_{0} \beta k_{0}^{2} / \hbar, e^{2} A_{0}^{2} \beta k_{0} / \hbar^{2}\right) \ll \hbar \Omega$. The first-order correction term $H_{F}^{(1)}$ vanishes for linearly polarized light. Other higher-order terms are too small to be considered here. Hence, the irradiated Hamiltonian can be simplified to an effective Hamiltonian as $H_{\text {eff }} \simeq H+H_{F}^{(0)}$, which can be diagonalized to obtain the Floquet energy spectrum as

$$
E_{k, s}=\frac{\hbar^{2} k^{2}}{2 m^{*}}+s \beta k \sqrt{\left[k^{2}-\alpha \sin (2 \theta)\right]^{2}+\alpha^{2} \cos ^{2}(2 \theta)},
$$

where $\tan \theta=k_{y} / k_{x}$. Note that the Floquet energy spectrum does not depend on the frequency of irradiation. The energy dispersion is now anisotropic for $\alpha \neq 0$. This is one of the interesting effects of irradiation. The corresponding eigenstates are given by $|k, s\rangle=\left[\begin{array}{ll}1 & i s e^{-i \chi}\end{array}\right]^{T} e^{i\left(k_{x} x+k_{y} y\right)} / \sqrt{2}$, where $\tan \chi=\left[\alpha \cos \theta-k^{2} \sin (3 \theta)\right] /\left[\alpha \sin \theta+k^{2} \cos (3 \theta)\right]$. Another interesting observation here is that the energy splitting between the two spin branches vanishes at the $\left(k_{r}, \theta_{r}\right)=$ $\left(\sqrt{\alpha}, \theta_{r}\right)$, when $\theta_{r}=(\pi / 4,5 \pi / 4)$, i.e., apart from $k=0$, a pair of additional spin-degeneracy points appears. Note that 

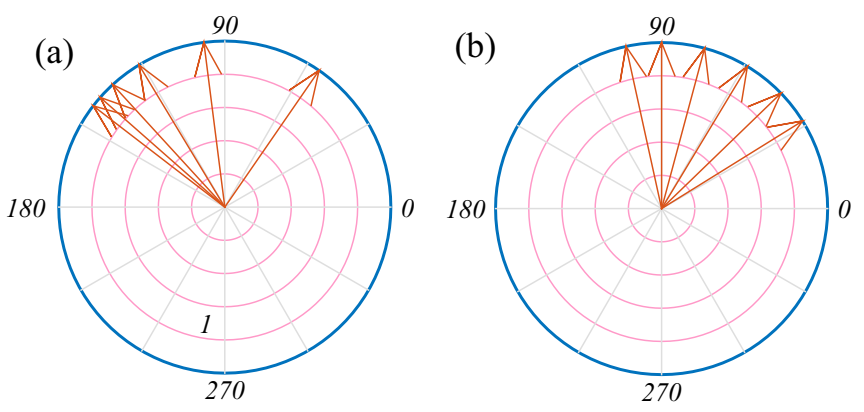

FIG. 2. Spin rotations in the $\left\langle S_{x}\right\rangle-\left\langle S_{y}\right\rangle$ plane are shown for (a) $k / k_{0}=0.3, \theta=\pi / 8$ and (b) $k / k_{0}=0.5, \theta=\pi / 8$ with increasing $\alpha / k_{0}^{2}$ from 0.05 to 0.3 in steps of 0.05 . Spin rotates in an anticlockwise direction with increasing $\alpha$. Both components of average spin are in units of $(3 / 2) \hbar$.

the locations of these degeneracy points do not depend on the strength of RSOC explicitly. The degeneracy points are shown by arrows in the Floquet energy spectrum, plotted in Fig. 1(b). If this degeneracy occurs at the Fermi level, then SHC exhibits a resonance, known as spin-Hall resonance (SHR) which was first discovered in a 2D electronic system with RSOC in the presence of a constant magnetic field [68]. The competition between Zeeman splitting and Rashba splitting causes such a degeneracy between two nearest Landau levels with opposite spin. The SHR phenomena was studied immediately in the 2DHG with $k^{3}$-RSOC [69]. However, note that in both works, the magnetic field plays the key role. In our work, we find that such a spin degeneracy can be achieved even without Landau levels, by applying linearly polarized light. Before going to discuss the SHC, we shall briefly discuss the effects of light on the average spin polarization.

Spin polarization. To examine the effects of irradiation on average spin polarization in projected spin space, we can evaluate different components of the average spin polarization as

$$
\left\langle k, s\left|\hat{S}_{x}\right| k, s\right\rangle=s \frac{k^{2} \sin (3 \theta)-\alpha \cos \theta}{\sqrt{k^{4}+\alpha^{2}-2 \alpha k^{2} \sin (2 \theta)}}
$$

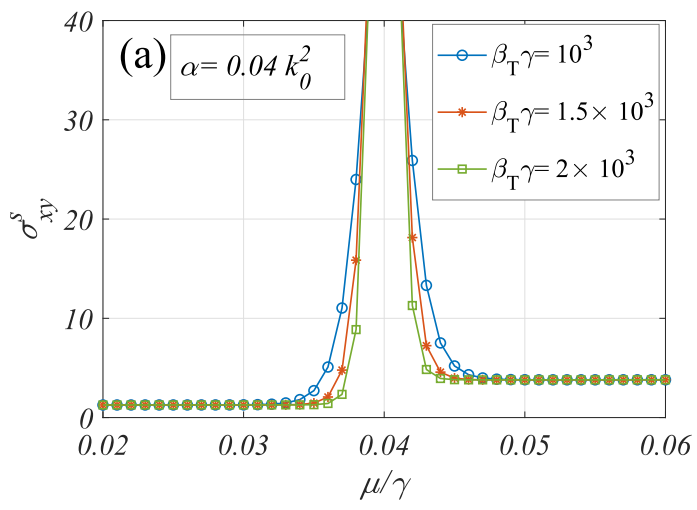

and

$$
\left\langle k, s\left|\hat{S}_{y}\right| k, s\right\rangle=s \frac{k^{2} \cos (3 \theta)+\alpha \sin \theta}{\sqrt{k^{4}+\alpha^{2}-2 \alpha k^{2} \sin (2 \theta)}},
$$

where $\hat{S}_{x, y}=(3 / 2) \hbar \hat{\sigma}_{x, y}$. It can be realized from the above expressions that away from the spin-degeneracy point, the spin polarization is significantly affected by the strength of irradiation $\alpha$. The effects of light on the average spin polarization are presented by a couple of compass plots in Fig. 2, showing anticlockwise rotation of the average spin with the increase of $\alpha$. We consider two sets of $(k, \theta)$, showing the sensitivity of spin rotation on momentum. It is to be noted that in the $k$-RSOC electron system [63], the effects of light on spin orientation or band structure can be observed only for circularly polarized light, whereas in our case the light is linearly polarized.

Spin-Hall resonance and discussion. We now investigate the SHC for the 2DHG in III-V semiconductor quantum wells with $k^{3}$-RSOC as a linear response to the applied in-plane electric field along the $x$ direction. The SHC can be evaluated by using the Kubo formula [38] as

$$
\begin{aligned}
\sigma_{x y}^{z}= & \frac{e \hbar}{L_{x} L_{y}} \sum_{k, s \neq s^{\prime}}\left[f\left(E_{k s^{\prime}}\right)-f\left(E_{k s}\right)\right] \\
& \times \frac{\operatorname{Im}\left[\left\langle k s\left|\hat{J}_{x}^{z}\right| k s^{\prime}\right\rangle\left\langle k s^{\prime}\left|\hat{v}_{y}\right| k s\right\rangle\right]}{\left(E_{k s^{\prime}}-E_{k s}\right)\left(E_{k s^{\prime}}-E_{k s}-i \epsilon\right)} .
\end{aligned}
$$

In the dc limit, $\epsilon$ is set to be zero. The system dimension is denoted by $L_{x} \times L_{y}$. The Fermi distribution function is denoted by $f\left(E_{k s}\right)$ for spin $s$. The spin-current operator is given by $\hat{J}_{x}^{z}=(3 \hbar / 4)\left\{\hat{\sigma}_{z}, \hat{v}_{x}\right\}$. Note that in general, one should proceed with the Floquet states $|k, s ; m\rangle$ in the Kubo formula in order to include the possible effects of the nearest Floquet sidebands. However, here we are only dealing with the zeroth-order correction (as the higher-order corrections vanish), hence the effects of Floquet sidebands can be safely ignored and we can proceed with the zeroth state $|k, s\rangle$. The velocity operators are given by $\hat{v}_{i}=\hbar^{-1} \partial H_{\text {eff }} / \partial k_{i}$, i.e.,

$$
\hat{v}_{x}=\frac{\hbar k_{x}}{m^{*}}+\frac{\beta}{\hbar}\left[3\left(k_{x}^{2}-k_{y}^{2}\right) \sigma_{y}-6 k_{x} k_{y} \sigma_{x}\right]+\frac{\alpha \beta}{\hbar} \sigma_{x}
$$

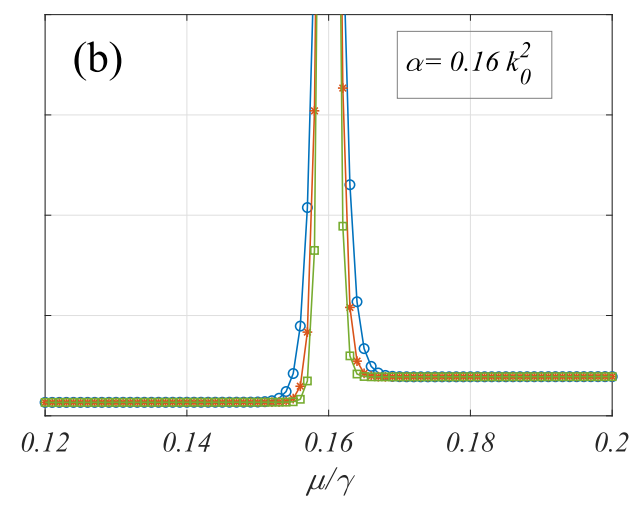

FIG. 3. SHC with variation of chemical potential for (a) $\alpha=0.04 k_{0}^{2}$ and (b) $\alpha=0.16 k_{0}^{2}$. The SHC (y axis) is normalized by $\sigma_{0}=$ $e\left(3 \hbar^{2} / 8 m^{*} \beta\right)$. The SHC exhibits resonance when the chemical potential crosses the spin-degeneracy points, i.e., when $\mu / \gamma=\alpha / k_{0}^{2}$. The strength of RSOC is taken as $\beta k_{0}^{3}=0.4$. The $\gamma$ is the same as in Fig. 1 . 
and

$$
\hat{v}_{y}=\frac{\hbar k_{y}}{m^{*}}+\frac{\beta}{\hbar}\left[3\left(k_{y}^{2}-k_{x}^{2}\right) \sigma_{x}-6 k_{x} k_{y} \sigma_{y}\right]+\frac{\alpha \beta}{\hbar} \sigma_{y} .
$$

With the computed matrix elements, we can rewrite the Kubo formula as

$$
\begin{aligned}
\sigma_{x y}^{z}= & \sigma_{0} \int \frac{d k}{(2 \pi)^{2}} \int \cos \theta d \theta\left[f\left(E_{k,+}\right)-f\left(E_{k,-}\right)\right] \\
& \times \frac{\cos \theta\left(\alpha^{2}+3 k^{4}\right)-\alpha k^{2}(\sin 3 \theta+3 \sin \theta)}{\left|\left(k^{2}-\alpha \sin 2 \theta\right)^{2}+\alpha^{2} \cos ^{2} 2 \theta\right|^{3 / 2}} .
\end{aligned}
$$

Here, $\sigma_{0}=(3 e / 8)\left(\hbar^{2} / m^{*} \beta\right)$. The SHC is evaluated numerically by using Eq. (10) and is plotted in Fig. 3. We normalize $k$ by $k_{0}$, a typical Fermi momentum corresponding to the hole density. The SHR occurs when the chemical potential becomes equal to $\alpha / k_{0}^{2}$, which is exactly the location of the spin-degeneracy points in the band dispersion (see Fig. 1). This can also be seen from the denominator of Eq. (10), which vanishes at the points $\left(k_{r}, \theta_{r 1}\right)=(\sqrt{\alpha}, \pi / 4)$ and $\left(k_{r}, \theta_{r 2}\right)=$ $(\sqrt{\alpha}, 5 \pi / 4)$, giving the resonance in the SHC. In each panel, three different but very low temperatures are considered as $\beta_{T} \gamma=10^{3}, 1.5 \times 10^{3}, 2 \times 10^{3}$, which only affect the broadening of the resonance peaks. Here, $\beta_{T}=1 / k_{B} T$, with $T$ being the absolute temperature and $k_{B}$ the Boltzmann constant. We also consider two different amplitudes of the light (i.e., location of the degeneracy points), which do not have any significant impact on the resonance phenomena, except for some minor effects on the temperature-dependent width of the resonance peak. We note that there is a jump in the SHC around the resonance which might be attributed to the asymmetric contribution of the velocity and spin-current matrix product to the total SHC [70]. We also check that away from the resonance, the SHC is not constant, but rather it weakly varies with the chemical potential. We use the usual definition of the spin-current operator, even though a modified definition had been used for studying SHR [71] in an electron system and it was noted that the new definition does not affect the SHR phenomena, except for an overall amplitude modification of the SHC.

Now we comment on the realistic parameter regime for such resonance to occur. At the degeneracy point, the Fermi momentum $k_{F}=\sqrt{2 \pi n_{d}}$ can be computed for a typical hole density [54] $\left(n_{d} \sim 2 \times 10^{15} \mathrm{~m}^{-2}\right)$ to be around $\sim 10^{8} \mathrm{~m}^{-1}$. On the other hand, the typical amplitude of the light field that is used in experiment is around $e A_{0} / \hbar \sim(1-10) \times 10^{8} \mathrm{~m}^{-1}$ corresponding to a photon energy of $\hbar \Omega=0.25 \mathrm{eV}$. This suggests that the degeneracy points, $k_{F}=\sqrt{3}\left(e A_{0} / \hbar\right)$, can be realized in a regular experimental setup [72].

Effects of a $k^{3}$-Dresselhaus type spin-orbit interaction. Now we briefly discuss the possible effects of irradiation in the presence of $k^{3}$-DSOC on a heavy-hole gas with RSOC. The DSOC arises due to the lack of bulk inversion symmetry in the system. This interaction was introduced by Loss in Ref. [44] in a study of spin relaxation and decoherence in quantum dots in the presence of a magnetic field. The spin transport was also studied including both the RSOC and DSOC terms [73]. The total Hamiltonian in the presence of RSOC and DSOC can be written as $H=\hbar^{2} k^{2} / 2 m^{*}+H_{R}+H_{D}$, where the DSOC term is given by $H_{D}=-(\lambda / 2)\left(k_{-} k_{+} k_{-} \sigma_{+}+k_{+} k_{-} k_{+} \sigma_{-}\right)$. Here, $\lambda$ is the strength of the DSOC. Following the same approach as for RSOC, the linearly polarized light-induced correction terms to the Hamiltonian $H$ are given by $-\lambda \alpha\left(k_{x} \sigma_{x}+\right.$ $\left.k_{y} \sigma_{y}\right) / 6-\lambda \alpha\left(k_{y} \sigma_{x}+k_{x} \sigma_{y}\right) / 3$. The energy eigenvalue in the presence of both the RSOC and the DSOC after incorporating the light-induced correction reads as $E_{k, s}=\hbar^{2} k^{2} / 2 m^{*}+$ $s \sqrt{h_{1}^{2}+h_{2}^{2}}$ with

$$
\begin{gathered}
h_{1}=-\beta k^{3} \sin (3 \theta)+\eta k \cos \theta-\frac{\alpha \lambda}{3} k \sin \theta, \\
h_{2}=\beta k^{3} \cos (3 \theta)+\eta k \sin \theta-\frac{\alpha \lambda}{3} k \cos \theta,
\end{gathered}
$$

where $\eta=\alpha(\beta-\lambda / 6)-\lambda k^{2}$. It can be seen that the spin splitting still vanishes at $k_{r}=\sqrt{\alpha(\beta-\lambda / 2) /(\beta+\lambda)}$ for $\theta_{r}=$ $(\pi / 4,5 \pi / 4)$. Therefore, the resonance phenomena observed in SHC should remain unaltered even in the presence of a weak DSOC in the hole system. The only noticeable point here is that now $k_{r}$ becomes sensitive to the strength of both RSOC and DSOC.

Conclusion. We have shown that linearly polarized light can induce anisotropy in the band structure of a twodimensional heavy-hole system with $k^{3}$-RSOC. Most remarkably, the spin-dependent band structure exhibits a pair of additional degeneracy points, which give rise to a resonance in the SHC. The appearance of the resonance in the spin-Hall transport may be a useful approach to achieve a giant SHC. We also discuss the fate of the resonance in the SHC in the presence of a structural-inversion-asymmetry-induced DSOC term and we confirm that the presence of this term only affects the location of the degeneracy points, while keeping the resonance phenomena unaffected. Finally, we would also like to comment that as the case of a two-dimensional electron system with $k$-RSOC, the application of circularly polarized light does open a gap between spin branches in our case. Therefore, the application of circularly polarized light will not be useful to achieve the spin-Hall resonance.

Acknowledgments. The authors thank Carsten Timm, Shyamal Biswas, Tarun K. Ghosh, and A. A. Zyuzin for useful discussions. A.B. acknowledges financial support by the Deutsche Forschungsgemeinschaft. S.F.I. acknowledges financial support by the Academy of Finland Grant No. 308339.
[1] T. Oka and H. Aoki, Photovoltaic Hall effect in graphene, Phys. Rev. B 79, 081406(R) (2009).

[2] J.-i. Inoue and A. Tanaka, Photoinduced spin Chern number change in a two-dimensional quantum spin Hall insulator with broken spin rotational symmetry, Phys. Rev. B 85, 125425 (2012).
[3] X. Zhai and G. Jin, Photoinduced topological phase transition in epitaxial graphene, Phys. Rev. B 89, 235416 (2014).

[4] M. Ezawa, Photoinduced Topological Phase Transition and a Single Dirac-Cone State in Silicene, Phys. Rev. Lett. 110, 026603 (2013). 
[5] P. M. Perez-Piskunow, G. Usaj, C. A. Balseiro, and L. E. F. Foa Torres, Floquet chiral edge states in graphene, Phys. Rev. B 89, 121401(R) (2014).

[6] J. Cayssol, B. Dóra, F. Simon, and R. Moessner, Floquet topological insulators, Phys. Status Solidi RRL 7, 101 (2013).

[7] N. H. Lindner, G. Refael, and V. Galitski, Floquet topological insulator in semiconductor quantum wells, Nat. Phys. 7, 490 (2011).

[8] Y.-G. Peng, C.-Z. Qin, D.-G. Zhao, Y.-X. Shen, X.-Y. Xu, M. Bao, H. Jia, and X.-F. Zhu, Experimental demonstration of anomalous Floquet topological insulator for sound, Nat. Commun. 7, 13368 (2016).

[9] H. Zhang, J. Yao, J. Shao, H. Li, S. Li, D. Bao, C. Wang, and G. Yang, Anomalous photoelectric effect of a polycrystalline topological insulator film, Sci. Rep. 4, 5876 (2014).

[10] Y. Wang, H. Steinberg, P. Jarillo-Herrero, and N. Gedik, Observation of Floquet-Bloch states on the surface of a topological insulator, Science 342, 453 (2013).

[11] A. López, Z. Z. Sun, and J. Schliemann, Floquet spin states in graphene under ac-driven spin-orbit interaction, Phys. Rev. B 85, 205428 (2012).

[12] A. Kundu, H. A. Fertig, and B. Seradjeh, Floquet-Engineered Valleytronics in Dirac Systems, Phys. Rev. Lett. 116, 016802 (2016).

[13] L. E. Golub, S. A. Tarasenko, M. V. Entin, and L. I. Magarill, Valley separation in graphene by polarized light, Phys. Rev. B 84, 195408 (2011).

[14] B. Dey and T. K. Ghosh, Photoinduced valley and electron-hole symmetry breaking in $\alpha-T_{3}$ lattice: The role of a variable Berry phase, Phys. Rev. B 98, 075422 (2018).

[15] M. Tahir, A. Manchon, and U. Schwingenschlögl, Photoinduced quantum spin and valley Hall effects, and orbital magnetization in monolayer $\mathrm{MoS}_{2}$, Phys. Rev. B 90, 125438 (2014)

[16] M. Tahir and U. Schwingenschlögl, Tunable thermoelectricity in monolayers of $\mathrm{MoS}_{2}$ and other group-VI dichalcogenides, New J. Phys. 16, 115003 (2014).

[17] Z. Yan and Z. Wang, Tunable Weyl Points in Periodically Driven Nodal Line Semimetals, Phys. Rev. Lett. 117, 087402 (2016).

[18] X.-X. Zhang, T.-T. Ong, and N. Nagaosa, Theory of photoinduced Floquet Weyl semimetal phases, Phys. Rev. B 94, 235137 (2016).

[19] R. Chen, B. Zhou, and D.-H. Xu, Floquet Weyl semimetals in light-irradiated type-II and hybrid line-node semimetals, Phys. Rev. B 97, 155152 (2018).

[20] A. Narayan, Tunable point nodes from line-node semimetals via application of light, Phys. Rev. B 94, 041409(R) (2016).

[21] M. Ezawa, Photoinduced topological phase transition from a crossing-line nodal semimetal to a multiple-Weyl semimetal, Phys. Rev. B 96, 041205(R) (2017).

[22] X. Zhou and G. Jin, Light-modulated $0-\pi$ transition in a silicene-based Josephson junction, Phys. Rev. B 94, 165436 (2016).

[23] U. Khanna, S. Rao, and A. Kundu, 0- $\pi$ transitions in a Josephson junction of an irradiated Weyl semimetal, Phys. Rev. B 95, 201115(R) (2017).

[24] A. Kundu and B. Seradjeh, Transport Signatures of Floquet Majorana Fermions in Driven Topological Superconductors, Phys. Rev. Lett. 111, 136402 (2013).
[25] D. E. Liu, A. Levchenko, and H. U. Baranger, Floquet Majorana Fermions for Topological Qubits in Superconducting Devices and Cold-Atom Systems, Phys. Rev. Lett. 111, 047002 (2013).

[26] Z. Yang, Q. Yang, J. Hu, and D. E. Liu, Dissipative Floquet Majorana Modes in Proximity-Induced Topological Superconductors, Phys. Rev. Lett. 126, 086801 (2021).

[27] D. T. Liu, J. Shabani, and A. Mitra, Floquet Majorana zero and $\pi$ modes in planar Josephson junctions, Phys. Rev. B 99, 094303 (2019).

[28] SK F. Islam and A. A. Zyuzin, Photoinduced interfacial chiral modes in threefold topological semimetal, Phys. Rev. B 100, 165302 (2019).

[29] M. Rodriguez-Vega, A. Kumar, and B. Seradjeh, Higher-order Floquet topological phases with corner and bulk bound states, Phys. Rev. B 100, 085138 (2019).

[30] A. K. Ghosh, G. C. Paul, and A. Saha, Higher order topological insulator via periodic driving, Phys. Rev. B 101, 235403 (2020).

[31] Y. Peng, Floquet higher-order topological insulators and superconductors with space-time symmetries, Phys. Rev. Res. 2, 013124 (2020).

[32] R. Winkler, Spin-Orbit Coupling Effects in Two-Dimensional Electron and Hole Systems, Springer Tracts in Modern Physics (Springer, Berlin, 2003).

[33] I. Žutić, J. Fabian, and S. Das Sarma, Spintronics: Fundamentals and applications, Rev. Mod. Phys. 76, 323 (2004).

[34] G. Engels, J. Lange, Th. Schäpers, and H. Lüth, Experimental and theoretical approach to spin splitting in modulation-doped $\mathrm{In}_{x} \mathrm{Ga}_{1-x} \mathrm{As} / \mathrm{InP}$ quantum wells for $B \rightarrow 0$, Phys. Rev. B 55, R1958 (1997).

[35] J. Nitta, T. Akazaki, H. Takayanagi, and T. Enoki, Gate Control of Spin-Orbit Interaction in an Inverted $\mathrm{In}_{0.53} \mathrm{Ga}_{0.47} \mathrm{As} / \mathrm{In}_{0.52} \mathrm{Al}_{0.48} \mathrm{As}$ heterostructure, Phys. Rev. Lett. 78, 1335 (1997).

[36] J. P. Heida, B. J. van Wees, J. J. Kuipers, T. M. Klapwijk, and G. Borghs, Spin-orbit interaction in a two-dimensional electron gas in a InAs/A1Sb quantum well with gate-controlled electron density, Phys. Rev. B 57, 11911 (1998).

[37] J. E. Hirsch, Spin Hall Effect, Phys. Rev. Lett. 83, 1834 (1999).

[38] J. Sinova, D. Culcer, Q. Niu, N. A. Sinitsyn, T. Jungwirth, and A. H. MacDonald, Universal Intrinsic Spin Hall Effect, Phys. Rev. Lett. 92, 126603 (2004).

[39] J. Sinova, S. O. Valenzuela, J. Wunderlich, C. H. Back, and T. Jungwirth, Spin Hall effects, Rev. Mod. Phys. 87, 1213 (2015).

[40] S. Murakami, N. Nagaosa, and S.-C. Zhang, Dissipationless quantum spin current at room temperature, Science 301, 1348 (2003).

[41] J.-I. Inoue, G. E. W. Bauer, and L. W. Molenkamp, Suppression of the persistent spin Hall current by defect scattering, Phys. Rev. B 70, 041303(R) (2004).

[42] O. V. Dimitrova, Vanishing spin-Hall conductivity in 2D disordered Rashba electron gas, arXiv:cond-mat/0405339.

[43] S. Murakami, Absence of vertex correction for the spin Hall effect in p-type semiconductors, Phys. Rev. B 69, 241202(R) (2004).

[44] D. V. Bulaev and D. Loss, Spin Relaxation and Decoherence of Holes in Quantum Dots, Phys. Rev. Lett. 95, 076805 (2005).

[45] F. Nichele, M. Kjaergaard, H. J. Suominen, R. Skolasinski, M. Wimmer, B.-M. Nguyen, A. A. Kiselev, W. Yi, M. Sokolich, M. J. Manfra, F. Qu, A. J. A. Beukman, L. P. Kouwenhoven, 
and C. M. Marcus, Giant Spin-Orbit Splitting in Inverted InAs/GaSb Double Quantum Wells, Phys. Rev. Lett. 118, 016801 (2017).

[46] R. Li, F. E. Hudson, A. S. Dzurak, and A. R. Hamilton, Pauli spin blockade of heavy holes in a silicon double quantum dot, Nano Lett. 15, 7314 (2015).

[47] J. Schliemann and D. Loss, Spin-Hall transport of heavy holes in III-V semiconductor quantum wells, Phys. Rev. B 71, 085308 (2005).

[48] B. Das, S. Datta, and R. Reifenberger, Zero-field spin splitting in a two-dimensional electron gas, Phys. Rev. B 41, 8278 (1990)

[49] X. F. Wang and P. Vasilopoulos, Magnetotransport in a two-dimensional electron gas in the presence of spin-orbit interaction, Phys. Rev. B 67, 085313 (2003).

[50] SK F. Islam and T. K. Ghosh, Zero-field spin splitting in a two-dimensional electron gas with the spin-orbit interaction revisited, J. Phys.: Condens. Matter 24, 035302 (2011).

[51] A. Mawrie, T. Biswas, and T. K. Ghosh, Magnetotransport properties of 2D fermionic systems with $k$-cubic Rashba spinorbit interaction, J. Phys.: Condens. Matter 26, 405301 (2014).

[52] A. Mawrie, S. Verma, and T. K. Ghosh, Electrical and thermoelectric transport properties of two-dimensional fermionic systems with $k$-cubic spin-orbit coupling, J. Phys.: Condens. Matter 29, 465303 (2017).

[53] A. Faridi, R. Asgari, and A. Langari, Magnetotransport of a two-dimensional electron gas with anisotropic Rashba interaction at the $\mathrm{LaAlO}_{3} / \mathrm{SrTiO}_{3}$ interface, Phys. Rev. B 98, 155442 (2018).

[54] H. Liu, E. Marcellina, A. R. Hamilton, and D. Culcer, Strong Spin-Orbit Contribution to the Hall Coefficient of TwoDimensional Hole Systems, Phys. Rev. Lett. 121, 087701 (2018).

[55] E. Marcellina, P. Bhalla, A. R. Hamilton, and D. Culcer, Signatures of quantum mechanical Zeeman effect in classical transport due to topological properties of two-dimensional spin-

$\frac{3}{2}$ holes, Phys. Rev. B 101, 121302(R) (2020).

[56] E. Marcellina, A. Srinivasan, D. S. Miserev, A. F. Croxall, D. A. Ritchie, I. Farrer, O. P. Sushkov, D. Culcer, and A. R. Hamilton, Electrical Control of the Zeeman Spin Splitting in Two-Dimensional Hole Systems, Phys. Rev. Lett. 121, 077701 (2018).

[57] J. H. Cullen, P. Bhalla, E. Marcellina, A. R. Hamilton, and D. Culcer, Generating a Topological Anomalous Hall Effect in a Nonmagnetic Conductor: An In-Plane Magnetic Field as a Direct Probe of the Berry Curvature, Phys. Rev. Lett. 126, 256601 (2021).
[58] L. Zhu, L. Zhu, M. Sui, D. C. Ralph, and R. A. Buhrman, Variation of the giant intrinsic spin Hall conductivity of Pt with carrier lifetime, Sci. Adv. 5, eaav8025 (2019).

[59] Y. Yen and G.-Y. Guo, Tunable large spin Hall and spin Nernst effects in the Dirac semimetals $\mathrm{Zr} X Y(X=\mathrm{Si}, \mathrm{Ge} ; Y=$ S, Se, Te), Phys. Rev. B 101, 064430 (2020).

[60] P. C. Lou, A. Katailiha, R. G. Bhardwaj, T. Bhowmick, W. P. Beyermann, R. K. Lake, and S. Kumar, Large spin Hall effect in Si at room temperature, Phys. Rev. B 101, 094435 (2020).

[61] C. L. Kane and E. J. Mele, Quantum Spin Hall Effect in Graphene, Phys. Rev. Lett. 95, 226801 (2005).

[62] M. Ezawa, Valley-Polarized Metals and Quantum Anomalous Hall Effect in Silicene, Phys. Rev. Lett. 109, 055502 (2012).

[63] T. Ojanen and T. Kitagawa, Photoinduced helical metal and magnetization in two-dimensional electron systems with spinorbit coupling, Phys. Rev. B 85, 161202(R) (2012).

[64] D. Hernangómez-Pérez, J. D. Torres, and A. López, Photoinduced electronic and spin properties of two-dimensional electron gases with Rashba spin-orbit coupling under perpendicular magnetic fields, Phys. Rev. B 102, 165414 (2020).

[65] J. M. Luttinger, Quantum theory of cyclotron resonance in semiconductors: General theory, Phys. Rev. 102, 1030 (1956).

[66] R. Winkler, Rashba spin splitting in two-dimensional electron and hole systems, Phys. Rev. B 62, 4245 (2000).

[67] A. Eckardt, Colloquium: Atomic quantum gases in periodically driven optical lattices, Rev. Mod. Phys. 89, 011004 (2017).

[68] S.-Q. Shen, M. Ma, X. C Xie, and F. C. Zhang, Resonant Spin Hall Conductance in Two-Dimensional Electron Systems with a Rashba Interaction in a Perpendicular Magnetic Field, Phys. Rev. Lett. 92, 256603 (2004).

[69] T. Ma and Q. Liu, Sign changes and resonance of intrinsic spin Hall effect in two-dimensional hole gas, Appl. Phys. Lett. 89, 112102 (2006).

[70] The jump can be understood by analyzing the integrand at $\theta_{r}=\pi / 4$ as $\left[f\left(E_{k,+}\right)-f\left(E_{k,-}\right)\right]\left(3 k^{2}-\alpha\right) \operatorname{sgn}\left(k^{2}-\alpha\right) / \mid k^{2}-$ $\left.\alpha\right|^{2}$, which is not symmetric across the resonance.

[71] Z. Wang and P. Zhang, Conversed spin Hall conductance in a two-dimensional electron gas in a perpendicular magnetic field, Phys. Rev. B 75, 233306 (2007).

[72] B. Kaestner, J. Wunderlich, T. Jungwirth, J. Sinova, K. Nomura, and A. H. MacDonald, Experimental observation of the spin-Hall effect in a spin-orbit coupled two-dimensional hole gas, Physica E: Low Dimens. Syst. Nanostruct. 34, 47 (2006).

[73] A. Wong and F. Mireles, Spin Hall and longitudinal conductivity of a conserved spin current in two-dimensional heavy-hole gases, Phys. Rev. B 81, 085304 (2010). 